\title{
Analysis of Ceramic Pattern Primitives Transform Operation
}

\author{
LI Busheng ${ }^{1}, \mathrm{HU}$ Jingfang ${ }^{2}$ \\ 1,2School of Information Engineering, Jingdezhen Ceramic Institute, Jiangxi, P.R.China \\ 1abulbs@163.com, 2idzhjf@163.com
}

Keywords: ceramic pattern; geometric transformation; Entity

Abstract. This paper analyzes the entity atomic operations include translation, scaling, rotation, mirror, waveform transform and vortex transformation mainly six geometric transformation, and according to the characteristics of ceramic pattern, has carried on the experiment to verify the analysis.

\section{Introduction}

Geometric arithmetic of image can change the spatial relationship between objects in an image. A geometric arithmetic requires two independent algorithms. The first, it need an algorithm to define space transform itself, describe how each pixel from the initial position to 'move' to terminate the position with it, namely each pixel 'move-ment'. Second, it also requires a gray interpolation algorithm. Because, the position coordinates of the input image (x,y) is an integer, but the position coordinates of the output image is not integer, the converse is also true.

In most applications, connectivity requirements keep curve features in the image of the continuity and the object. An unconstrained spatial transform algorithm is likely to break line and broken images. In general we use mathematical methods to describe the spatial relationship between the input and output image point. The general definition for geometric arithmetic:

$$
g(x, y)=f\left(x^{\prime}, y^{\prime}\right)=f[a(x, y), b(x, y)]
$$

Wherein, $f(x, y)$ represents the input image, $g(x, y)$ represents the output image. The function $a(x$, $y)$ and $b(x, y)$ only describes the spatial transformation. If they are continuous, then the continuous relationship will be maintained in the image [1].

\section{The Geometric Transformation of Graphic}

The first requirement of geometric transformation is to run an algorithm which moves the pixel from a position to another position. We introduce several common geometric transformations.

Through the previous introduction, we are ready for the geometric transformation of the image. In equation (1), if

$$
a(x, y)=x^{\prime} \text { And } b(x, y)=y^{\prime}
$$

Then we get an identity arithmetic simply copy f to $\mathrm{G}$ without any changes.

\section{A Translation}

If makes the $a(x, y)=x+x 0$, and $b(x, y)=y+y 0$ then it get a translation arithmetic, the point $(x 0, y 0)$ is translated to the origin, and the feature points in the image move $\sqrt{\left(x_{0}^{2}+y_{0}^{2}\right)}$. Using the 
homogeneous coordinate expression way, we can think the plane $x y$ is a plane which $\mathrm{Z}=0$ in the three-dimensional space $x, y, z$. And the equation (2) expressed in concise matrix as follows:

$$
\left(\begin{array}{l}
a(x, y) \\
b(x, y) \\
1
\end{array}\right)=\left(\begin{array}{ccc}
1 & 0 & x 0 \\
0 & 1 & y 0 \\
0 & 0 & 1
\end{array}\right)\left(\begin{array}{l}
x \\
y \\
1
\end{array}\right)
$$

\section{B Scaling}

If the $a(x, y)=x / c, B(x, y)=y / d$, it will make the image magnified $c$ times in the $X$ direction, magnified $\mathrm{D}$ times in the direction of $\mathrm{Y}$ axis. The origins of image keep still when the image is magnified.

$$
\left(\begin{array}{l}
a(x, y) \\
b(x, y) \\
1
\end{array}\right)=\left(\begin{array}{ccc}
1 / c & 0 & x 0 \\
0 & 1 / d & y 0 \\
0 & 0 & 1
\end{array}\right)\left(\begin{array}{l}
x \\
y \\
1
\end{array}\right)
$$

\section{Mirror}

If the $\mathrm{c}=1$, it will produce a $\mathrm{Y}$ axis symmetric image $\mathrm{a}(\mathrm{x}, \mathrm{y})=-\mathrm{x}, \mathrm{b}(\mathrm{x}, \mathrm{y})=\mathrm{y}$.

$$
\left(\begin{array}{l}
a(x, y) \\
b(x, y) \\
1
\end{array}\right)=\left(\begin{array}{ccc}
-1 & 0 & 0 \\
0 & 1 & 0 \\
0 & 0 & 1
\end{array}\right)\left(\begin{array}{l}
x \\
y \\
1
\end{array}\right)
$$

Similarly, also have the same properties for $\mathrm{D}$ and $\mathrm{X}$.

$$
\left(\begin{array}{l}
a(x, y) \\
b(x, y) \\
1
\end{array}\right)=\left(\begin{array}{ccc}
1 & 0 & 0 \\
0 & -1 & 0 \\
0 & 0 & 1
\end{array}\right)\left(\begin{array}{l}
x \\
y \\
1
\end{array}\right)
$$

\section{Rotation}

Let $a(x, y)=x \cos (\theta)-y \sin (\theta)$ and $b(x, y)=x \sin (\theta)+y \cos (\theta)$, the image is to be rotated clockwise $\theta$ degrees about its center.

$$
\left(\begin{array}{l}
a(x, y) \\
b(x, y) \\
1
\end{array}\right)=\left(\begin{array}{ccc}
\cos (\theta) & -\sin (\theta) & 0 \\
\sin (\theta) & \cos (\theta) & 0 \\
0 & 0 & 1
\end{array}\right)\left(\begin{array}{l}
x \\
y \\
1
\end{array}\right)
$$

\section{E Waveform}

If the waveform transform is level, then we makes

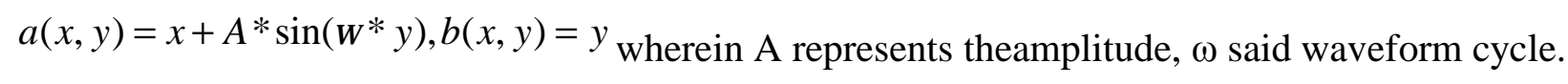

$$
\left(\begin{array}{l}
a(x, y) \\
b(x, y) \\
1
\end{array}\right)=\left(\begin{array}{ccc}
1 & A^{*} \sin \left(\omega^{*} y\right) / y & 0 \\
0 & 1 & 0 \\
0 & 0 & 1
\end{array}\right)\left(\begin{array}{l}
x \\
y \\
1
\end{array}\right)
$$

But if it is a vertical waveform transform, then 


$$
\left(\begin{array}{l}
a(x, y) \\
b(x, y) \\
1
\end{array}\right)=\left(\begin{array}{ccc}
0 & 1 & 0 \\
1 & A * \sin (\omega * x) / x & 0 \\
0 & 0 & 1
\end{array}\right)\left(\begin{array}{l}
x \\
y \\
1
\end{array}\right)
$$

\section{F Vortex}

If we set the coordinates of the vortex points for the $(x, y)$, the scroll angle for the $\theta$, it will be :

$$
\begin{aligned}
& a(x, y)=x^{*} \cos \theta-y^{*} \sin \theta+x 0 *(1-\cos \theta)+y 0 * \sin \theta \\
& b(x, y)=x^{*} \sin \theta+y^{*} \cos \theta+y 0 *(1-\cos \theta)-x 0 * \sin \theta
\end{aligned}
$$

\section{Gray Interpolation Algorithm}

The second requirement of the Geometric transformation of is to run the gray interpolation algorithm. In the input image $\mathrm{f}(\mathrm{x}, \mathrm{y})$, gray value is defined only at integer positions $(\mathrm{x}, \mathrm{y})$. However, in equation (1), the gray value of $\mathrm{G}$ ( $\mathrm{x}, \mathrm{y}$ ) determine by the value of the non integer coordinates $\mathrm{F}(\mathrm{x}, \mathrm{y})$ in general .Therefore, if the geometrical arithmetic as a mapping from $\mathrm{F}$ to $\mathrm{g}$, a pixel in $\mathrm{F}$ will be mapped to the locations between several pixels, the converse is also true. In order to discussion convenient, we specified all pixels are located just at the integer coordinates of the sampling grid.

Commonly used two kinds of interpolation, one is the nearest neighbor interpolation, the other is the bilinear interpolation. Bilinear interpolation can produce more satisfactory results compared with the nearest neighbor interpolation, therefore, we use the bilinear interpolation method in this paper .The principle of bilinear interpolation to the following [2]:

Let $\mathrm{f}(\mathrm{x}, \mathrm{y})$ as a function of two variables, the value of the vertex in the unit square is known, and there have a linear equation:

$$
f(x, y)=a x+b y+c x y+d
$$

We can define a hyperbolic parabolic and four known points have been fitted by the bilinear equation. The four coefficients from a to D must be selected by the $f(x, y)$ of the four apexes. Firstly, it will calculate two above apexes with linear interpolation, we can get:

$$
f(x, 0)=f(0,0)+x[f(1,0)-f(0,0)]
$$

By the same steps, we calculate two down apexes with linear interpolation, we can get:

$$
f(x, 1)=f(0,1)+x[f(1,1)-f(0,1)]
$$

At last, we calculate vertical direction with linear interpolation:

$$
f(x, y)=f(x, 0)+y[f(x, 0)-f(x, 0)]
$$

\section{Experimental Verification}

Now we list the atomic operation key code of the level waveform as an example:

\{

widthAfterS=(int)(curCell.pCell->GetWidth()+2*fabs(A)+0.5);

heightAfterS=curCell.pCell->GetHeight(); 


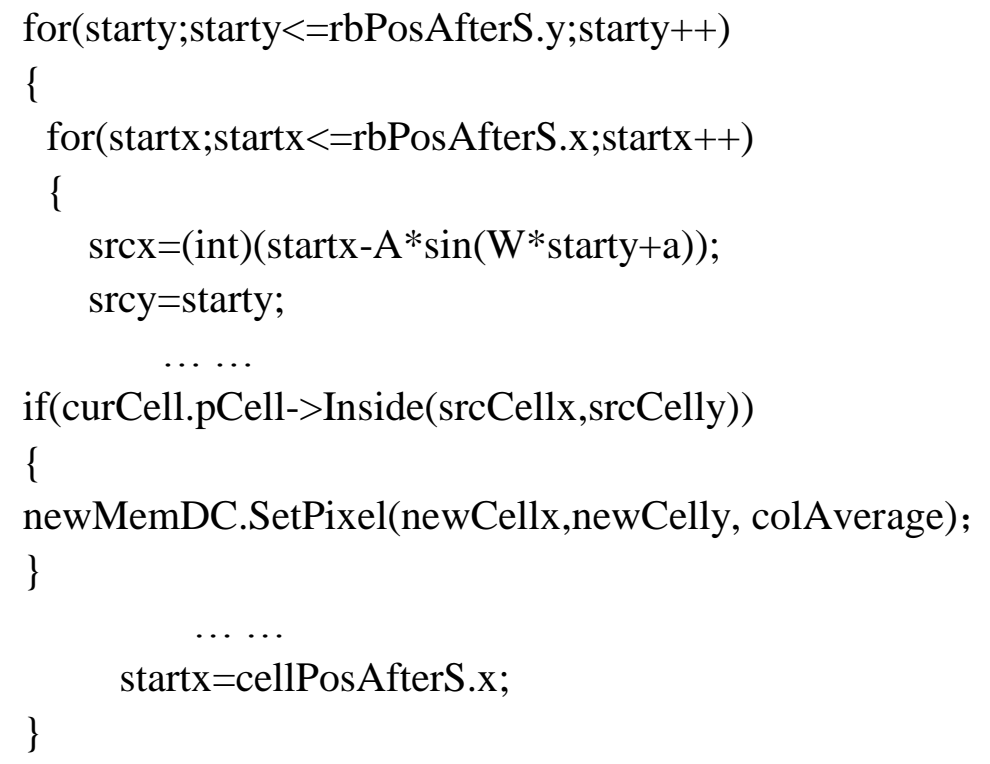

Figure 1 is the two sides successive pattern pattern obtained by two horizontal waveform effect, figure 2 is a suitable pattern after two times the effect of vortex transform.

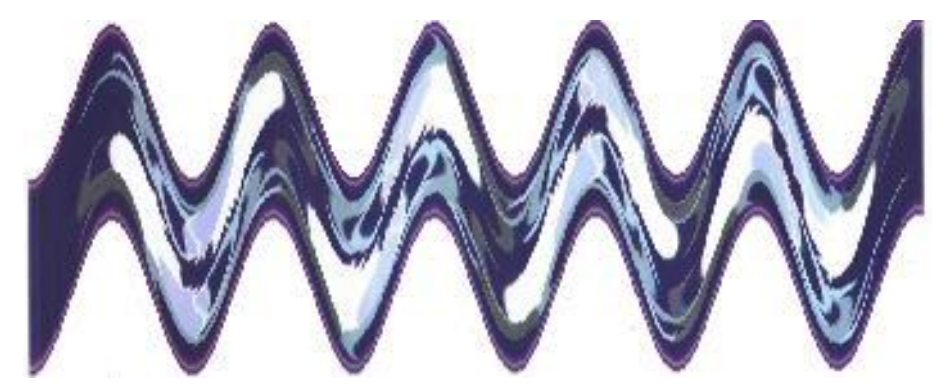

Fig. 1 horizontal waveform transformation

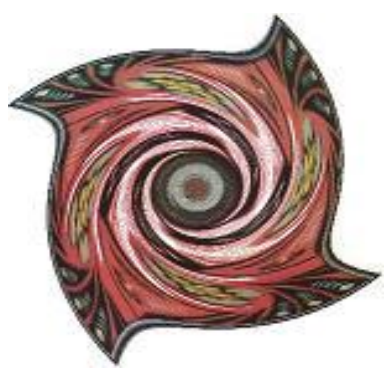

Fig. 2 swirl transformation

\section{Conclusion}

This paper analyzes the six main algorithms of pattern geometry transformation in translation, scaling, rotation, mirror, waveform transform and vortex transform, and analysis and interpolation algorithm of gray value, and then through the experiment validated the ceramic pattern, the results show that the design of ceramic pattern can use geometric transformation algorithm widely, so as to facilitate the rapid building ceramic pattern.

\section{References}

[1] Yang Shuying. VC++ image processing program design. Beijing Northern Jiaotong University press. 2003:79 85.

[2] Liu Xiaozhou, Tu defense. Application of Wavelet bilinear interpolation in optical remote sensing image. Journal of Graduate University of Chinese Academy of Sciences. 2003.20 (1):39 42.

[3] Li S.Tan Y.L. One rapid segmentation algorithm for quasi-circular fruits. Recent advances in computer science and information engineering. 2012, 128: 287-293.

[4] Mesas-Carrascosa F.J., Castillejo-Gonzalez I.L., Orden M.S.Dl.et al. Real-time mobile phone application to supper land policy. Computers and electronics in agriculture. 2012, 85(2012): 109-111. 